\title{
CONOCIMIENTO DE LOS FUTUROS EDUCADORES SOCIALES DE LAS HERRAMIENTAS WEB 2.0
}

\section{KNOWLEDGE THE FUTURE SOCIAL EDUCATORS ABOUT 2.0 TOOLS}

\author{
Begoña E. Sampedro Requena \\ bsampedro@uco.es \\ Dra. Verónica Marín Díaz \\ vmarin@uco.es
}

Universidad de Córdoba. Facultad de Ciencias de la Educación. Departamento de Educación. Avda. San Alberto Magno s/n 14004

Córdoba (España)

\begin{abstract}
Hoy las capacidades formativas y profesionales del educador social se han visto modificadas, entre otros aspectos, por la incorporación de las TIC a estos ámbitos. Presentamos a continuación el estudio realizado sobre el conocimiento que poseen los estudiantes de Educación Social de la Universidad Pablo de Olavide sobre las herramientas Web 2.0 que más se emplean hoy. Como resultados principales destacar que estos tienen un conocimiento elevado de las redes sociales frente a las wikis que las desconocen, no existiendo diferencias en torno al sexo, pero si al curso académico cursado, siendo los estudiantes de primer año los que más herramientas conocen.

Palabras clave: educador social, conocimiento, herramientas 2.0, Web 2.0, formación.
\end{abstract}

Today the social educator's training capacity and professional skills have been changed by the new trends of ITC, amongst other factors. We present a research on the knowledge that Social Education Bachelor's degree students of the University of Pablo de Olavide have about the most widely used web 2.0 tools, nowadays. The main results show they have a good knowledge about social networks but they do not know wikis, there are not statistical differences regarding sex, but there is a difference concerning the academic course they are taking, being first year students the ones who know more web 2.0 tools.

Keywords: Social Education Teacher, Knowledge, Web 2.0 tools, training. 


\section{Introducción.}

La Web 2.0 ha revolucionado los procesos de enseñanza-aprendizaje como indican Grodecka, Pata y Väljataga (2008, p.10), pues según estos autores «esta innovación tecnológica posee el potencial de cambiar la forma de aprender». En este sentido, no debemos obviar los actuales puntos de vista de corte pedagógico, los cuales se basan en conceptos sociales y colaborativos (Freire, 2007), como señala Esteve (2009, p. 61) las herramientas Web 2.0 «generan espacios de comunicación idóneos para el desarrollo de algunas de las habilidades y, sobre todo, actitudes de un nuevo tipo de alfabetización tecnológica crítica, colaborativa y creativa».

En esta línea, los educadores sociales, como agentes educativos en el ámbito de la educación formal, deben adquirir este conocimiento tecnológico, tanto en su formación inicial como en el ejercicio de su profesión, pues entre las competencias específicas que se deberán fomentar en la instrucción de estos profesionales se encuentran las «capacidades cognitivas como adquisición de conocimientos, tanto a nivel de constructo y funcionamiento como a nivel de aplicabilidad en lo contextos educativos y sociales» (Sevillano, 2010, p. 19).

En el libro blanco para el título de Grado en Pedagogía y Educación Social elaborado por la ANECA (2005) ya se contemplaba esta perspectiva, dado que entre las competencias transversales con carácter instrumental para el educador social se expone la utilización de las TIC en el ámbito de estudio y contexto profesional, la cual es interpretada por este organismo en los siguientes términos «es la capacidad para utilizar las TIC como una herramienta para la expresión y la comunicación, para el acceso a fuentes de información, como medio de archivo de datos y documentos, para tareas de presentación, para el aprendizaje, la investigación y el trabajo cooperativo» (ANECA, 2005, p. 143).

En este sentido, López Meneses, Llorent y Fernández Márquez (2013, p. 12) señalan que «las nuevas tendencias tecnológicas emergentes son recursos muy valiosos para la construcción del conocimiento en los procesos de aprendizaje, propiciando la reformulación de metodologías socioconstructivistas e investigadoras», de ahí que pensemos que las distintas experiencias didácticas realizadas, por estos autores, con el alumnado del Grado de Educador Social, hayan puesto de manifiesto la relevancia y necesidad de analizar estos aspectos.

Las aportaciones de todos estos autores (López Meneses, Llorent \& Fernández Márquez, 2013; Sevillano, 2010) ponen de relieve que el desarrollo de la competencia tecnológica, entendida esta como el conocimiento y la aplicabilidad de la misma en contextos sociales, posibilita un rendimiento superior y de mayor calidad en el proceso de enseñanza - aprendizaje en las instituciones educativas.

Cabero (2009) señala: pero lo verdaderamente importante desde mi punto de vista no es lo cuantitativo de las tecnologías, sino lo cualitativo, qué repercute para que los jóvenes se relacionen de otra manera, que procesen la información de forma distinta, que cambien el concepto de tiempo, que formen redes sociales con otras reglas del juego y de manera digital y, lo que a nosotros más nos interesa, que aborden el proceso de formación e instrucción desde otras perspectivas (p.15).

En efecto, la forma de aprender de los estudiantes ha variado y, por lo tanto, se debe 
dotar a todos los agentes educativos, incluido el educador social, en su formación inicial de las herramientas necesarias para afrontar su labor profesional en el ámbito de la educación, dado que la misma se extiende fuera de las aulas (Herrera, 2010).

Martínez (2002) considera que así las cosas, las nuevas demandas y retos planteados por la recién implantada Sociedad de la Información establecen una clara vinculación entre las TIC y la educación, pues resulta ya evidente la necesidad de abordarlas como un nuevo área de conocimiento ineludible, cuyo aprendizaje, además, no pasa sólo por reconocer y manejar sus aplicaciones prácticas, sino por intentar comprender el cambio de modelo social que alumbra su implementación (citado en Martínez 2004, p. 242).

Este cambio en el modelo social provocado por las tecnologías es un factor a tener en cuenta por los profesionales educativos, posiblemente con mayor relevancia para el educador social debido a los ámbitos de intervención en los que ejerce su labor (Gómez Gutiérrez, 2000).

En definitiva, considerando el papel del educador social en el ámbito educativo formal y las tendencias tecnológicas presentes, tanto en la sociedad como en las instituciones escolares, es necesario el estudio del conocimiento que poseen estos agentes educativos a lo largo de su formación inicial de las herramientas Web 2.0, dado que las mismas favorecen entornos más prácticos, eficaces y útiles para la creación y producción de aplicaciones y contenidos (Cabero, 2009; Grodecka, Pata \& Väljataga, 2008), sin embargo, existen pocos antecedentes teóricos actuales a este respecto, consecuentemente creemos que el abordaje de este estudio cobra mayor relevancia.

\section{Metodología.}

El objetivo principal de esta investigación es determinar el grado de conocimiento que posee el alumnado de la titulación de Grado en Educador Social, impartida en la Universidad Pablo de Olavide (Sevilla) sobre once herramientas Web 2.0, seleccionadas a través del ranking establecido por el Center for Learnig \& Performance Technologies en el año 2012 y revisado posteriormente en 2013 (http://o4lpt.co.uk/top100tools).

Las hipótesis propuestas a partir del mencionado objetivo, se agrupan en estadísticas y de investigación, estas últimas formuladas en términos de interrogante, y las primeras en forma de análisis matemático.

- Interrogantes: ¿Qué grado de conocimiento posee el alumnado de la titulación de Grado de Educador Social de la Universidad Pablo de Olavide de Sevilla sobre determinadas herramientas Web 2.0?

- Estadísticas: Existen diferencias estadísticamente significativas entre el promedio del conocimiento de una batería de 11 herramientas Web 2.0 en función del sexo del alumnado; el curso al que se adscriben; la procedencia; el tipo de vivienda; el dispositivo de acceso a Internet; la tipología de conexión empleada y la frecuencia semanal y diaria de conectividad.

Las variables propuestas para el objetivo que se presenta en este artículo son once, deteniéndonos en el estudio de la dimensión referida al conocimiento, y siendo estas las seleccionadas a través del ranking anteriormente indicado: entornos de aprendizaje virtuales, blogs, redes sociales y educativas, editores de video y de imágenes, buscadores de imágenes y buscadores de video, buscadores de texto y de presentaciones, wiki y foros. 
Este trabajo de investigación se basa en un paradigma positivista cuya finalidad reside en la explicación de la realidad observada; la metodología utilizada con carácter, exclusivamente, cuantitativa, se fundamenta a través de un método ex post facto, que tal y como indica Mateo (2012, p.196) proporciona, entre otros, «diseños descriptivos y comparativos», los cuales se han aplicado en este trabajo.

\subsection{Muestra.}

El problema que planteamos va dirigido a la población formada por el alumnado del Grado de Educador Social de la Universidad Pablo de Olavide (Sevilla), para la selección de la muestra se ha considerado el tamaño y la representatividad (Morales, 2012), siendo un total de 188 estudiantes con un error muestral de $\pm 3 \%$ y un nivel de confianza del $95 \%$, los cuales fueron encuestados durante el curso académico 2012/2013. La característica descriptiva general de esta muestra es ser estudiante del Grado de Educador Social de la Universidad Pablo de Olavide, situándose la media de edad en los 22.23 años. Atendiendo al sexo, encontramos un $82.9 \%$ de mujeres y un $17.1 \%$ de hombres; asimismo, el $54.3 \%$ proceden de ciudad y el $45.7 \%$ de pueblo; residiendo habitualmente el $52.8 \%$ en el domicilio familiar y el $47.2 \%$ en piso de estudiantes. En lo que se refiere al curso en el que se encuentran durante la realización de este estudio, el $27.7 \%$ es alumnado de primero; el $26.6 \%$ de segundo; el $24.4 \%$ de tercero y el $21.3 \%$ cursan cuarto de grado. Por otro lado, el $42.2 \%$ emplean una red pública y el $57.8 \%$ privada para su conexión a Internet, para esta conectividad el $25.1 \%$ utilizan el móvil, el $68.8 \%$ el Pc y solo el $6 \%$ la Tablet; siendo la frecuencia semanal de conexión del 13.6\% dos o tres días, el 33.2\% cuatro o cinco y el $53.3 \%$ todos los días de la semana, mientras que si nos referimos al acceso a la red por horas al día, el $37.2 \%$ lo hacen una o dos horas, el $41.2 \%$ entre tres o cuatro y el $21.6 \%$ más de cinco.

\subsection{Instrumento.}

Respecto al instrumento post hoc diseñado, este se divide en tres grandes dimensiones. El primero de ellos hace referencia a los datos socio-demográficos, el segundo al conocimiento y uso académico y personal, y el tercero, relativo a los 6 ámbitos de intervención del educador social en el campo educativo. Su medición es igual para los dos últimos bloques, escala Likert de cinco opciones ( $1=$ Desconozco, $2=$ Conocimiento bajo, 3= Conocimiento medio, 4= Conocimiento alto y $5=$ Conocimiento máximo), sin embargo varía la significación de las escalas de intervalos para cada dimensión.

Teniendo en cuenta que el instrumento utilizado es de elaboración propia se le debe dotar de validez y fiabilidad, realizando diferentes análisis y procesos. Para la validez de contenido se ha procedido a realizar un juicio de expertos, a través del «Panel Internacional de Investigación en Tecnología Educativa (PI2TE)» de la asociación EDUTEC, (http://gte2.uib.es/ panel). Estos han valorado positivamente la pertinencia, la secuenciación, la claridad y el diseño de las dimensiones, y de forma indiferente, la longitud del cuestionario. Asimismo, para la validez de constructo se ha realizado un análisis factorial exploratorio con un método de extracción de máxima verosimilitud y rotación varimax, el cálculo previo del índice de Kaiser- Meyer y Olkin 
$(\mathrm{KMO}=.886)$ y la prueba de esfericidad de Bartlett $(\mathrm{p}<.001)$, indican significatividad y viabilidad en la ejecución del mismo. La agrupación de los ítems del cuestionario refrenda una estructura de diversos factores, concretamente para los reactivos de este artículo un factor, cuya varianza extraída explica el $69 \%$ de los casos.

En lo que se refiere a la fiabilidad a través del enfoque de consistencia interna, el resultado obtenido al aplicar el procedimiento de Alfa de Cronbach ha sido de .934, este mismo análisis replicado para las nueve dimensiones estudiadas se posiciona en un intervalo de .888 para el uso personal y .978 para la valoración y utilidad en el ámbito de intervención 6 , de forma más determinada para la dimensión conocimiento el valor obtenido ha sido .960. Por otro lado, al realizar el coeficiente de homogeneidad corregido a los 164 ítems, los valores de todo ellos se acercan más a uno que a cero, lo cual refleja una fiabilidad alta de los ítems expresados en el cuestionario. Por último, buscando la fortaleza en el carácter unidimensional de la prueba (índice de fiabilidad) se ha realizado un análisis de la capacidad de discriminación de los reactivos a través de la prueba estadística $\mathrm{t}$ de Student (para muestras independientes) entre las medias de los grupos establecidos, revelando un alto poder de discriminación en todos los ítems (valores de significatividad $\mathrm{p}<.001)$.

\subsection{Estrategias de análisis de datos.}

El análisis de los datos recogidos tras la aplicación del cuestionario es de carácter eminentemente cuantitativo siendo, en primer lugar, de carácter descriptivo básico de las variables (medidas de tendencia central y medidas de dispersión). Seguidamente, se ha procedido a realizar estudios inferenciales atendiendo a criterios de clasificación como el sexo, el curso, la procedencia, el tipo de vivienda, el dispositivo de acceso a Internet, la tipología de conexión y la frecuencia semanal y diaria de conectividad, los mismos se han realizado con el programa estadístico SPSS (v.20).Para constatar los resultados obtenidos en la aplicación de los Análisis de Varianza (ANOVA) se realizaron los procedimientos post hoc como las pruebas de Scheffé y Bonferroni, ya que como indica Morales (2011) se debe implementar el análisis de Scheffé cuando no hay hipótesis previas o existen diferencias entre el tamaño de los grupos de la muestra. En nuestro estudio es la segunda premisa la que se cumple. Este mismo autor señala, que la prueba de Bonferroni se basa en la modificación del nivel de significación en función del número de comparaciones a realizar (Morales, 2011).

\section{Resultados.}

Los datos obtenidos en esta investigación, relativos a la dimensión conocimiento de 11 herramientas Web 2.0 se exponen en la Tabla 1, la cual presenta el promedio de todo el alumnado de la titulación de Grado en Educador Social de la Universidad Pablo de Olavide de Sevilla durante el curso académico 2012/2013.

Como se observa, el alumnado participante posee un conocimiento alto de las redes sociales y educativas y de los buscadores de texto (ambos con $\bar{x}=4.05$ ), mientras que indican un conocimiento medio-alto de los blogs y los foros ( $\bar{x}=3.77$ uno y otro), de los buscadores de imágenes $(\bar{x}=3.69)$, de los entornos de aprendizaje virtuales $(\bar{x}=3.62)$, de los buscadores de video $(\bar{x}=3.60)$, de los buscadores de presentaciones $(\bar{x}=3.59)$ y 


\begin{tabular}{|c|c|c|c|}
\hline & $\mathbf{N}$ & Media & $\mathbf{s}$ \\
\hline Conocimiento entornos de aprendizaje virtuales & 188 & 3.62 & 0.945 \\
\hline Conocimiento blogs & 188 & 3.77 & 0.908 \\
\hline Conocimiento redes sociales y educativas & 188 & 4.05 & 0.827 \\
\hline Conocimiento editores de video & 188 & 3.57 & 0.989 \\
\hline Conocimiento editores de imágenes & 188 & 3.50 & 0.970 \\
\hline Conocimiento buscadores de imágenes & 188 & 3.69 & 0.976 \\
\hline Conocimiento buscadores de video & 188 & 3.60 & 0.958 \\
\hline Conocimiento buscadores de texto & 188 & 4.05 & 0.796 \\
\hline Conocimiento buscadores de presentaciones & 188 & 3.59 & 0.949 \\
\hline Conocimiento wiki & 188 & 2.83 & 0.881 \\
\hline Conocimiento foros & 188 & 3.77 & 0.861 \\
\hline
\end{tabular}

Tabla 1.Conocimiento de la muestra de determinadas herramientas Web 2.0.

de los editores de video $(\bar{x}=3.57)$. Asimismo, expresan un conocimiento medio de los editores de imágenes $(\bar{x}=3.50)$ y un conocimiento bajo-medio de la wiki $(\bar{x}=2.83)$.

Procediendo a establecer diferencias estadísticamente significativas atendiendo al sexo del alumnado, se ha aplicado una prueba t de Student (n.s.= .05) cuyos resultados han revelado que esta variable no provoca contraste alguno en esta dimensión.

Asimismo, debido a la adscripción de este grupo de estudiantes en diferentes cursos, hemos realizado un ANOVA (n.s.= .05) obteniendo los siguientes valores representativos que se muestran en la Tabla 2.

Los resultados que arroja este análisis de varianza reflejan que el alumnado que cursa primero tiene un mayor conocimiento en todas las herramientas mostradas en la Tabla 2 que el resto de los cursos, de forma más concreta:
- En los entornos de aprendizaje, comparado con el resto de alumnos que se adscriben a segundo [Scheffé: Primero (I) Segundo $(J) p=.026$ y Bonferroni $\mathrm{p}=.014]$, tercero [Scheffé: Primero (I) - Tercero (J) p< .001 y Bonferroni $\mathrm{p}<.001] \mathrm{y}$, cuarto [Scheffé: Primero (I) - Cuarto (J) p = .001 y Bonferroni $\mathrm{p}<.001]$ el conocimiento es más alto en el alumnado de primero.

- El blog es una de las herramientas Web 2.0 en la que los estudiantes de primero poseen un conocimiento más alto en relación a los que cursan cuarto [Scheffé: Primero (I) - Cuarto (J) p <. 001 y Bonferroni $\mathrm{p}<.001]$; mientras que no podemos afirmar los mismos resultados para los cursos de segundo y tercero dado que los valores del análisis de la tasa de error no son reveladores.

Los estudiantes pertenecientes al primer curso asientan un conocimiento alto de las redes sociales y educativas con 


\begin{tabular}{|c|c|c|c|c|c|}
\hline $\begin{array}{l}\text { Conocimiento de algunas } \\
\text { herramientas Web } 2.0\end{array}$ & Curso & $\mathbf{N}$ & Media & $\mathbf{s}$ & $\begin{array}{l}\text { F y p de los grupos } \\
\text { favorables }\end{array}$ \\
\hline \multirow{4}{*}{$\begin{array}{c}\text { Entornos de aprendizaje } \\
\text { virtuales }\end{array}$} & Primero & 52 & 4.13 & 0.802 & \multirow{4}{*}{$\begin{array}{l}\mathrm{F}=9.165 \text { y } \mathrm{p}<.001 \\
\text { favorable a primero }\end{array}$} \\
\hline & Segundo & 50 & 3.60 & 0.774 & \\
\hline & Tercero & 46 & 3.29 & 1.137 & \\
\hline & Cuarto & 40 & 3.38 & 0.795 & \\
\hline \multirow{4}{*}{ Blogs } & Primero & 52 & 4.11 & 0.769 & \multirow{4}{*}{$\begin{array}{l}\mathrm{F}=6.232 \text { y } \mathrm{p}<.001 \\
\text { favorable a primero }\end{array}$} \\
\hline & Segundo & 50 & 3.77 & 0.757 & \\
\hline & Tercero & 46 & 3.76 & 1.069 & \\
\hline & Cuarto & 40 & 3.33 & 0.874 & \\
\hline \multirow{4}{*}{ Redes sociales y educativas } & Primero & 52 & 4.35 & 0.805 & \multirow{4}{*}{$\begin{array}{l}\mathrm{F}=8.098 \text { y } \mathrm{p}<.001 \\
\text { favorable a primero }\end{array}$} \\
\hline & Segundo & 50 & 4.25 & 0.653 & \\
\hline & Tercero & 46 & 3.71 & 0.901 & \\
\hline & Cuarto & 40 & 3.83 & 0.762 & \\
\hline \multirow{4}{*}{ Buscadores de video } & Primero & 52 & 3.87 & 0.891 & \multirow{4}{*}{$\begin{array}{l}\mathrm{F}=2.877 \text { y } \mathrm{p}=.037 \\
\text { favorable a primero }\end{array}$} \\
\hline & Segundo & 50 & 3.65 & 0.968 & \\
\hline & Tercero & 46 & 3.49 & 1.046 & \\
\hline & Cuarto & 40 & 3.33 & 0.846 & \\
\hline \multirow{4}{*}{ Buscadores de texto } & Primero & 52 & 4.33 & 0.727 & \multirow{4}{*}{$\begin{array}{l}\mathrm{F}=4.984 \text { y } \mathrm{p}=.002 \\
\text { favorable a primero }\end{array}$} \\
\hline & Segundo & 50 & 4.10 & 0.634 & \\
\hline & Tercero & 46 & 3.96 & 0.937 & \\
\hline & Cuarto & 40 & 3.74 & 0.767 & \\
\hline \multirow{4}{*}{ Foros } & Primero & 52 & 4.04 & 0.699 & \multirow{4}{*}{$\begin{array}{l}\mathrm{F}=3.259 \text { y } \mathrm{p}=.023 \\
\text { favorable a primero }\end{array}$} \\
\hline & Segundo & 50 & 3.77 & 0.921 & \\
\hline & Tercero & 46 & 3.73 & 0.874 & \\
\hline & Cuarto & 40 & 3.50 & 0.890 & \\
\hline
\end{tabular}

Tabla 2.ANOVA del conocimiento de algunas herramientas Web 2.0 en función de los cursos a los que se adscribe el alumnado.

respecto a los de tercero [Scheffé: Primero (I) - Tercero $(\mathrm{J})$ con $\mathrm{p}=0,001$ y Bonferroni $\mathrm{p}<$ .001] y a los de cuarto [Scheffé: Primero (I) Cuarto $(\mathrm{J}) \mathrm{p}=.018$ y Bonferroni: Primero $(\mathrm{I})-$ Cuarto $(\mathrm{J})$ con $\mathrm{p}=.009$ ]; en tanto que la comparación con el nivel de segundo no presenta significación en los valores de Scheffé y Bonferroni.
La herramienta Web 2.0 buscadores de texto es más conocida por la muestra de primero que por la de cuarto [Scheffé: Primero (I) - Cuarto (J) p = .004 y Bonferroni $\mathrm{p}=.001]$; por el contrario las comparaciones múltiples realizadas con tercero y segundo no arrojan datos relevantes.

Píxel-Bit. Revista de Medios y Educación. No 47 Julio 2015. ISSN: 1133-8482. e-ISSN: 2171-7966. doi: http://dx.doi.org/10.12795/pixelbit.2015.i47.03 
El alumnado de primero señala un conocimiento más alto en los foros que el de cuarto [Scheffé: Primero (I) - Cuarto (J) p = .026 y Bonferroni $\mathrm{p}=.014]$; en cambio no podemos afirmar los mismos resultados para los cursos de segundo y tercero dado que los valores del análisis de la tasa de error no son indicativos.

Igualmente, los estudiantes de primero tienen un conocimiento medio de los buscadores de video en comparación con los de cuarto [Scheffé: Primero (I) - Cuarto (J) p $=.050$ y Bonferroni $\mathrm{p}=.038 \mathrm{]}$ el resultado de esta comparativa no se puede extender a los cursos de segundo y tercero, considerando los valores emitidos por los análisis de Scheffé y Bonferroni.

El resto de herramientas Web 2.0, editores de video, editores y buscadores de imágenes, buscadores de presentaciones y wiki no revelan diferencias significativas del conocimiento en relación al curso al que se adscriben, en otros términos, el análisis de varianza no refleja resultados relevantes.

Por otra parte, el análisis de medias entre la dimensión del conocimiento de algunas herramientas Web 2.0 y la procedencia de la muestra a través de la prueba inferencial t de Student (n.s. $=.05)$ provoca los siguientes resultados significativos:

Las personas que habitan en los pueblos indican un conocimiento medio - alto ( = 3.84) en la tipología de herramienta Web 2.0 «buscadores de imágenes» $(\mathrm{t}=-1.990 \mathrm{y}$ $\mathrm{p}=.048)$ que las personas que residen habitualmente en la ciudad $(=3.56)$.

Asimismo, el cotejo entre medias revela que el conocimiento en «buscadores de video» $(\mathrm{t}=-2.008 \mathrm{y} \mathrm{p}=.046)$ es medio-alto en las personas que viven de forma continuada en los pueblos $(=3.75)$.
El resto de herramientas Web 2.0 estudiadas en esta investigación no presentan resultados significativos relativos a la media en función de la procedencia de la muestra.

Asimismo, al tratar de establecer diferencias estadísticamente significativas atendiendo a la residencia habitual del alumnado, aplicando para la misma una prueba $\mathrm{t}$ de Student (n.s.= .05), los resultados han reflejado que el tipo de vivienda no es una variable que provoque diferenciación.

Considerando, en esta ocasión, el dispositivo que emplea habitualmente la muestra participante, hemos efectuado un ANOVA (n.s.=.05) obteniendo los siguientes datos distintivos que se muestran en la Tabla 3.

Este análisis de contraste de igualdad de medias para el factor dispositivo de conexión refleja un alto conocimiento de determinadas herramientas Web 2.0 (ver Tabla 3) en el alumnado que emplea habitualmente el móvil frente a los que emplean el Pc, además la comparativa entre este y la tablet no arrojan resultados significativos en las pruebas post hoc aplicadas, las cuales son:

Los blog en comparación con los del Pc en todas sus modalidades ordenador de mesa, portátil o netbook [Scheffé: Móvil (I) $\operatorname{Pc}(\mathrm{J}) \mathrm{p}<.001$ y Bonferroni $\mathrm{p}<.001]$.

- Las redes sociales y educativas [Scheffé: Móvil $(\mathrm{I})-\mathrm{Pc}(\mathrm{J}) \mathrm{p}<.001$ y Bonferroni $\mathrm{p}<.001]$.

En los buscadores de imágenes [Scheffé: Móvil (I) - Pc (J) p <.001 y Bonferroni $\mathrm{p}<.001]$.

- Los buscadores de texto [Scheffé: Móvil (I) - Pc (J) p = .001 y Bonferroni $\mathrm{p}<$ .001].

. $\quad$ En la wiki [Scheffé: Móvil (I) - Pc (J) p $<.001$ y Bonferroni $\mathrm{p}<.001]$. 


\begin{tabular}{|c|c|c|c|c|c|}
\hline $\begin{array}{l}\text { Conocimiento de algunas } \\
\text { herramientas Web } 2.0\end{array}$ & $\begin{array}{l}\text { Acceso a } \\
\text { Internet }\end{array}$ & $\mathbf{N}$ & Media & $\mathbf{s}$ & F y p de los grupos favorables \\
\hline \multirow{3}{*}{$\begin{array}{c}\text { Entornos de aprendizaje } \\
\text { virtuales }\end{array}$} & Móvil & 47 & 3.98 & 0.892 & \multirow{3}{*}{$\begin{array}{c}\mathrm{F}=7.162 \text { y } \mathrm{p}=.001 \text { favorable al } \\
\text { acceso a través del móvil }\end{array}$} \\
\hline & $\mathrm{Pc}$ & 130 & 3.45 & 0.939 & \\
\hline & Tablet & 11 & 4.00 & 0.739 & \\
\hline \multirow{3}{*}{ Blogs } & Móvil & 47 & 4.18 & 0.825 & \multirow{3}{*}{$\begin{array}{c}\mathrm{F}=9.157 \text { y } \mathrm{p}<.001 \text { favorable al } \\
\text { acceso a través del móvil }\end{array}$} \\
\hline & $\mathrm{Pc}$ & 130 & 3.59 & 0.896 & \\
\hline & Tablet & 11 & 4.08 & 0.793 & \\
\hline \multirow{3}{*}{ Redes sociales y educativas } & Móvil & 47 & 4.50 & 0.580 & \multirow{3}{*}{$\begin{array}{c}\mathrm{F}=11.247 \text { y } \mathrm{p}<.001 \text { favorable al } \\
\text { acceso a través móvil }\end{array}$} \\
\hline & $\mathrm{Pc}$ & 130 & 3.88 & 0.858 & \\
\hline & Tablet & 11 & 4.08 & 0.827 & \\
\hline \multirow{3}{*}{ Editores de video } & Móvil & 47 & 4.04 & 0.903 & \multirow{3}{*}{$\begin{array}{c}\mathrm{F}=12.970 \text { y } \mathrm{p}<.001 \text { favorable al } \\
\text { acceso a través del móvil y la } \\
\text { tablet }\end{array}$} \\
\hline & $\mathrm{Pc}$ & 130 & 3.34 & 0.960 & \\
\hline & Tablet & 11 & 4.17 & 0.718 & \\
\hline \multirow{3}{*}{ Editores de imágenes } & Móvil & 47 & 4.00 & 0.782 & \multirow{3}{*}{$\begin{array}{c}\mathrm{F}=13.504 \text { y } \mathrm{p}<.001 \text { favorable al } \\
\text { acceso a través del móvil y la } \\
\text { tablet }\end{array}$} \\
\hline & $\mathrm{Pc}$ & 130 & 3.27 & 0.970 & \\
\hline & Tablet & 11 & 4.00 & 0.739 & \\
\hline \multirow{3}{*}{ Buscadores de imágenes } & Móvil & 47 & 4.24 & 0.822 & \multirow{3}{*}{$\begin{array}{c}\mathrm{F}=13.965 \text { y } \mathrm{p}<.001 \text { favorable al } \\
\text { acceso a través móvil }\end{array}$} \\
\hline & $\mathrm{Pc}$ & 130 & 3.46 & 0.947 & \\
\hline & Tablet & 11 & 4.00 & 0.953 & \\
\hline \multirow{3}{*}{ Buscadores de video } & Móvil & 47 & 4.10 & 0.839 & \multirow{3}{*}{$\begin{array}{c}\mathrm{F}=13.477 \text { y } \mathrm{p}<.001 \text { favorable al } \\
\text { acceso a través del móvil y la } \\
\text { tablet }\end{array}$} \\
\hline & $\mathrm{Pc}$ & 130 & 3.38 & 0.940 & \\
\hline & Tablet & 11 & 4.08 & 0.669 & \\
\hline \multirow{3}{*}{ Buscadores de texto } & Móvil & 47 & 4.40 & 0.639 & \multirow{3}{*}{$\begin{array}{c}\mathrm{F}=8.009 \text { y } \mathrm{p}<.001 \text { favorable al } \\
\text { acceso a través móvil }\end{array}$} \\
\hline & $\mathrm{Pc}$ & 130 & 3.91 & 0.812 & \\
\hline & Tablet & 11 & 4.25 & 0.754 & \\
\hline \multirow{3}{*}{ Buscadores de presentaciones } & Móvil & 47 & 3.98 & 0.915 & \multirow{3}{*}{$\begin{array}{c}\mathrm{F}=7.730 \text { y } \mathrm{p}=.001 \text { favorable al } \\
\text { acceso a través móvil }\end{array}$} \\
\hline & $\mathrm{Pc}$ & 130 & 3.42 & 0.921 & \\
\hline & Tablet & 11 & 3.92 & 0.900 & \\
\hline Wiki & Móvil & 47 & 4.24 & 0.744 & $\mathrm{~F}=9.729$ y $\mathrm{p}<.001$ favorable al \\
\hline
\end{tabular}

Tabla 3. ANOVA del conocimiento de algunas herramientas Web 2.0 en función del dispositivo de conexión. 
Y, los foros [Scheffé: Móvil (I) - Pc (J) $\mathrm{p}=.001$ y Bonferroni $\mathrm{p}<.001]$.

De igual forma, el alumnado que emplea frecuentemente el móvil para su acceso a Internet posee un conocimiento medio- alto de las siguientes herramientas en comparación con los del Pc (sin que la comparativa con la tablet refleje significación):

De los entornos de aprendizaje virtuales [Scheffé: Móvil (I) $-\mathrm{Pc}$ (J) p=.003 y Bonferroni $\mathrm{p}=.002]$.

$\mathrm{Y}, \mathrm{de}$ los buscadores de presentaciones [Scheffé: Móvil (I) - Pc (J) p $=.001$ y Bonferroni $\mathrm{p}=.001]$.

Por otro lado, en función del empleo habitual de los dispositivos móvil y tablet en comparación con el Pc se refleja un conocimiento alto por parte de los estudiantes de tres de las once herramientas Web 2.0 ( $\mathrm{sin}$ embargo no se refleja relevancia entre la comparativa de estos dos utensilios móviles como indica las pruebas de Scheffé y Bonferroni aplicadas), siendo estas:

Los editores de video [Scheffé: Móvil (I) $-\mathrm{Pc}(\mathrm{J}) \mathrm{p}<.001$ y Bonferroni $\mathrm{p}<.001]$ y el contraste tablet y Pc [Scheffé: Tablet (I) - Pc (J) $\mathrm{p}=.014$ y Bonferroni $\mathrm{p}=.011$ ].

Los editores de imágenes [Scheffé: Móvil (I) - Pc (J) p <.001 y Bonferroni $\mathrm{p}<$ .001 y Scheffé: Tablet (I) - Pc (J) p= .032 y Bonferroni $\mathrm{p}=.027)(\mathrm{J})]$.

Y, los buscadores de video [Scheffé: Móvil (I) - Pc (J) p <.001 y Bonferroni p < .001 y Scheffé: Tablet (I) - Pc (J) p $=.037$ y Bonferroni $\mathrm{p}=.031$ ].

Al tratar de establecer diferencias estadísticamente significativas atendiendo a los días de la semana de conexión a Internet, aplicando para la misma una ANOVA (n.s.= .05), los resultados arrojados son (ver Tabla 4).
El alumnado que se conecta a Internet todos los días posee un conocimiento alto de tres de las once herramientas Web 2.0 considerando la comparativa con las otras frecuencias de conexión (las pruebas post hoc aplicadas refrendan estos resultados), las cuales son:

Los entornos de aprendizaje virtuales [Scheffé: Todos los días (I) - 2 o 3 días (J) p< .001 y Bonferroni $p<.001 ;$ Scheffé: Todos los días (I) -4 o 5 días (J) $p<.001$ y Bonferroni $p<$ $.001]$.

Los buscadores de texto [Scheffé: Todos los días (I) - 2 o 3 días (J) p< .001 y Bonferroni $\mathrm{p}<.001$; Scheffé: Todos los días (I) -4 o 5 días $(\mathrm{J}) \mathrm{p}=.001$ y Bonferroni $\mathrm{p}=$ $.001]$.

- Y, la wiki [Scheffé: Todos los días (I) 2 o 3 días (J) $\mathrm{p}<.001$ y Bonferroni $\mathrm{p}<.001$; Scheffé: Todos los días (I) -4 o 5 días (J) p .001 y Bonferroni $\mathrm{p}<.001]$.

Así mismo, los estudiantes que se conectan a Internet todos los días poseen un conocimiento medio-alto de determinadas herramientas Web 2.0 en comparación a los que acceden en las otras frecuencias, tal y como señalan las pruebas de Scheffé y Bonferroni aplicadas, siendo estas:

Los editores de video [Scheffé: Todos los días (I) - 2 o 3 días (J) p < .001 y Bonferroni $\mathrm{p}<.001$;Scheffé: Todos los días (I) - 4 o 5 días $(J) \mathrm{p}<.001$ y Bonferroni $\mathrm{p}<.001]$.

Los editores de imágenes [Scheffé: Todos los días (I) - 2 o 3 días (J) $\mathrm{p}<.001$ y Bonferroni $\mathrm{p}<.001 ;$ Scheffé: Todos los días (I) -4 o 5 días (J) $\mathrm{p}<.001$ y Bonferroni $\mathrm{p}<$ $.001]$.

Los buscadores de video [Scheffé: Todos los días (I) - 2 o 3 días (J) p $<.001$ y Bonferroni $\mathrm{p}<.001 ;$ Scheffé: Todos los días (I) -4 o 5 días (J) $\mathrm{p}=.002$ y Bonferroni $\mathrm{p}=$ $.001]$. 


\begin{tabular}{|c|c|c|c|c|c|}
\hline $\begin{array}{c}\text { Conocimiento de algunas } \\
\text { herramientas Web } 2.0\end{array}$ & $\begin{array}{l}\text { Acceso por } \\
\text { semana }\end{array}$ & $\mathbf{N}$ & Media & $\mathbf{s}$ & $\begin{array}{c}\text { F y p de los grupos } \\
\text { favorables }\end{array}$ \\
\hline \multirow{3}{*}{$\begin{array}{c}\text { Entornos de aprendizaje } \\
\text { virtuales }\end{array}$} & 2 o 3 días & 25 & 2.89 & 0.892 & \multirow{3}{*}{$\begin{array}{c}\mathrm{F}=25.348 \text { y } \mathrm{p}<.001 \\
\text { favorable al acceso todos los } \\
\text { días }\end{array}$} \\
\hline & 4 o 5 días & 62 & 3.30 & 0.859 & \\
\hline & Todos los días & 101 & 4.00 & 0.828 & \\
\hline \multirow{3}{*}{ Blogs } & 2 o 3 días & 25 & 3.04 & 0.898 & \multirow{3}{*}{$\begin{array}{c}\mathrm{F}=17.695 \text { y } \mathrm{p}<.001 \\
\text { favorable al acceso todos los } \\
\text { días y a } 4 \text { o } 5 \text { días }\end{array}$} \\
\hline & 4 o 5 días & 62 & 3.61 & 0.782 & \\
\hline & Todos los días & 101 & 4.06 & 0.860 & \\
\hline \multirow{3}{*}{$\begin{array}{c}\text { Redes sociales y } \\
\text { educativas }\end{array}$} & 2 o 3 días & 25 & 3.22 & 0.847 & \multirow{3}{*}{$\begin{array}{c}\mathrm{F}=23.453 \text { y } \mathrm{p}<.001 \\
\text { favorable al acceso todos los } \\
\text { días y a } 4 \text { o } 5 \text { días }\end{array}$} \\
\hline & 4 o 5 días & 62 & 3.97 & 0.723 & \\
\hline & Todos los días & 101 & 4.31 & 0.735 & \\
\hline \multirow{3}{*}{ Editores de video } & 2 o 3 días & 25 & 2.96 & 0.854 & \multirow{3}{*}{$\begin{array}{c}\mathrm{F}=19.102 \text { y } \mathrm{p}<.001 \\
\text { favorable al acceso todos los } \\
\text { días }\end{array}$} \\
\hline & 4 o 5 días & 62 & 3.23 & 0.856 & \\
\hline & Todos los días & 101 & 3.93 & 0.953 & \\
\hline \multirow{3}{*}{ Editores de imágenes } & 2 o 3 días & 25 & 2.89 & 0.847 & \multirow{3}{*}{$\begin{array}{c}\mathrm{F}=17.010 \text { y } \mathrm{p}<.001 \\
\text { favorable al acceso todos los } \\
\text { días }\end{array}$} \\
\hline & 4 o 5 días & 62 & 3.21 & 0.832 & \\
\hline & Todos los días & 101 & 3.84 & 0.952 & \\
\hline \multirow{3}{*}{ Buscadores de imágenes } & 2 o 3 días & 25 & 3.04 & 0.854 & \multirow{3}{*}{$\begin{array}{c}\mathrm{F}=10.464 \text { y } \mathrm{p}<.001 \\
\text { favorable al acceso todos los } \\
\text { días y } 4 \text { o } 5 \text { días }\end{array}$} \\
\hline & 4 o 5 días & 62 & 3.58 & 0.786 & \\
\hline & Todos los días & 101 & 3.92 & 1.030 & \\
\hline \multirow{3}{*}{ Buscadores de video } & 2 o 3 días & 25 & 2.93 & 0.781 & \multirow{3}{*}{$\begin{array}{c}\mathrm{F}=15,621 \text { y } \mathrm{p}<0,001 \\
\text { favorable al acceso todos los } \\
\text { días }\end{array}$} \\
\hline & 4 o 5 días & 62 & 3.39 & 0.782 & \\
\hline & Todos los días & 101 & 3.91 & 0.981 & \\
\hline \multirow{3}{*}{ Buscadores de texto } & 2 o 3 días & 25 & 3.52 & 0.893 & \multirow{3}{*}{$\begin{array}{c}\mathrm{F}=14.985 \text { y } \mathrm{p}<.001 \\
\text { favorable al acceso todos los } \\
\text { días }\end{array}$} \\
\hline & 4 o 5 días & 62 & 3.86 & 0.742 & \\
\hline & Todos los días & 101 & 4.30 & 0.706 & \\
\hline \multirow{3}{*}{$\begin{array}{l}\text { Buscadores de } \\
\text { presentaciones }\end{array}$} & 2 o 3 días & 25 & 2.96 & 0.706 & \multirow{3}{*}{$\begin{array}{c}\mathrm{F}=13.331 \text { y } \mathrm{p}<.001 \\
\text { favorable al acceso todos los } \\
\text { días }\end{array}$} \\
\hline & 4 o 5 días & 62 & 3.39 & 0.875 & \\
\hline & Todos los días & 101 & 3.87 & 0.947 & \\
\hline Wiki & 2 o 3 días & 25 & 3.41 & 0.888 & $\mathrm{~F}=15.033 \mathrm{y} \mathrm{p}<.001$ \\
\hline
\end{tabular}

Tabla 4. ANOVA del conocimiento de algunas herramientas Web 2.0 en función de los días a la semana que se conecta los participantes.

Píxel-Bit. Revista de Medios y Educación. No 47 Julio 2015. ISSN: 1133-8482. e-ISSN: 2171-7966. doi: http://dx.doi.org/10.12795/pixelbit.2015.i47.03 
Los buscadores de presentaciones [Scheffé: Todos los días (I) - 2 o 3 días (J) p< .001 y Bonferroni $\mathrm{p}<.001$;Scheffé: Todos los días $(\mathrm{I})-4$ o 5 días $(\mathrm{J}) \mathrm{p}=.004$ y Bonferroni $\mathrm{p}=$ $.003]$.

Y, los foros [Scheffé: Todos los días (I) -2 o 3 días $(\mathrm{J}) \mathrm{p}=.011$ y Bonferroni $\mathrm{p}=$ .008; Scheffé: Todos los días (I) - 4 o 5 días (J) $\mathrm{p}=.005$ y Bonferroni $\mathrm{p}=.004]$.

Por otra parte, la muestra participante que accede todos los días a Internet tiene un conocimiento alto y la que accede 4 o 5 días un conocimiento medio de dos de las once herramientas Web 2.0 de este estudio (existiendo valores significativos en el análisis de la tasa de error entre todas las comparativas), estas son:

Los blog [Scheffé: Todos los días (I) 2 o 3 días $(\mathrm{J}) \mathrm{p}<.001$ y Bonferroni $\mathrm{p}<$ .001;Scheffé: Todos los días (I) - 4 o 5 días (J) $\mathrm{p}=.003$ y Bonferroni $\mathrm{p}=.002]$; asimismo, los que se conectan 4 o 5 días [Scheffé: 4 o 5 días (I) -2 o 3 días $(\mathrm{J}) \mathrm{p}=.014$ y Bonferroni $\mathrm{p}=$ .010].

Las redes sociales y educativas [Scheffé: Todos los días (I) - 2 o 3 días (J) p< .001 y Bonferroni $\mathrm{p}<.001$;Scheffé: Todos los días (I) -4 o 5 días (J) $\mathrm{p}=.016$ y Bonferroni $\mathrm{p}=.012]$; de la misma forma, el cotejo entre $4 \mathrm{o}$ 5 días [Scheffé: 4 o 5 días (I) - 2 o 3 días (J) p $<.001$ y Bonferroni $\mathrm{p}<.001]$.

Por último, el conocimiento medio-alto en los buscadores de imágenes se proporciona más en los participantes que se conectan todos los días a Internet en comparación con los que lo hacen 2 o 3 días [Scheffé: Todos los días (I) - 2 o 3 días $(\mathrm{J}) \mathrm{p}<.001$ y Bonferroni $\mathrm{p}<.001$ ], por el contrario no podemos afirmar los mismos resultados para el acceso a Internet de 4 o 5 días dado que los valores del análisis de la tasa de error no son indicativos. Mientras que los que se conectan 4 o 5 días frente a la conectividad de 2 o 3 gozan de un conocimiento medio mayor favorable a los primeros [Scheffé: 4 o 5 días $(\mathrm{I})-2$ o 3 días $(\mathrm{J}) \mathrm{p}=.043$ y Bonferroni $\mathrm{p}$ $<.037]$.

Por lo que se refiere al establecimiento de diferencias estadísticamente significativas atendiendo al conocimiento de los participantes en función de las horas al días de conexión a Internet hemos ejecutado para la misma un ANOVA (n.s.=.05), los resultados más relevantes se muestran en la Tabla 5.

El alumnado que se conecta a Internet más de 5 horas al día posee un conocimiento alto de determinadas herramientas Web 2.0 considerando la comparación con las otras frecuencias de conexión horaria (las pruebas post hoc aplicadas refrendan estos resultados), las cuales son:

Los blog [Scheffé: Más de 5 horas (I) -1 o 2 horas $(\mathrm{J}) \mathrm{p}<.001$ y Bonferroni $\mathrm{p}<$ .001;Scheffé: Más de 5 horas (I) - Entre 3 o 4 horas $(\mathrm{J}) \mathrm{p}=.003$ y Bonferroni $\mathrm{p}=.002]$.

Las redes sociales y educativas [Scheffé: Más de 5 horas (I) - 1 o 2 horas (J) $\mathrm{p}<.001$ y Bonferroni $\mathrm{p}<.001$, sin significación en la comparativa más de 5 horas y entre 3 o 4 horas].

Los buscadores de imágenes [Scheffé: Más de 5 horas (I) - 1 o 2 horas $(\mathrm{J}) \mathrm{p}<.001 \mathrm{y}$ Bonferroni $\mathrm{p}<.001$;Scheffé: Más de 5 horas (I) - Entre 3 o 4 horas $(\mathrm{J}) \mathrm{p}=.003$ y Bonferroni $\mathrm{p}=.002]$.

- $\quad \mathrm{Y}$, los buscadores de presentaciones [Scheffé: Más de 5 horas (I) - 1 o 2 horas (J) $\mathrm{p}<.001$ y Bonferroni $\mathrm{p}<.001 ;$ Scheffé: Más de 5 horas (I) - Entre 3 o 4 horas $(\mathrm{J}) \mathrm{p}=.004 \mathrm{y}$ Bonferroni $\mathrm{p}=.003$ ].

Por otro lado, los estudiantes que se conectan más de 5 horas diarias a Internet poseen un conocimiento alto y los que acceden entre 3 o 4 horas un conocimiento 


\begin{tabular}{|c|c|c|c|c|c|}
\hline $\begin{array}{c}\text { Conocimiento de algunas } \\
\text { herramientas Web 2.0 }\end{array}$ & Acceso por horas al día & $\mathbf{N}$ & Media & $\mathbf{s}$ & $\begin{array}{c}\text { F y p de los grupos } \\
\text { favorables }\end{array}$ \\
\hline \multirow{3}{*}{$\begin{array}{c}\text { Entornos de aprendizaje } \\
\text { virtuales }\end{array}$} & 1 o 2 horas & 70 & 3.26 & 0.812 & \multirow{3}{*}{$\begin{array}{c}\mathrm{F}=11.847 \text { y } \mathrm{p}<.001 \\
\text { favorable al acceso de } \\
\text { más de } 5 \text { horas y entre } \\
3 \text { o } 4 \text { horas }\end{array}$} \\
\hline & Entre 3 o 4 horas & 77 & 3.71 & 0.949 & \\
\hline & Más de 5 horas & 41 & 4.07 & 0.936 & \\
\hline \multirow{3}{*}{ Blogs } & 1 o 2 horas & 70 & 3.45 & 0.779 & \multirow{3}{*}{$\begin{array}{c}\mathrm{F}=14.490 \text { y } \mathrm{p}<.001 \\
\text { favorable al acceso de } \\
\text { más de } 5 \text { horas. }\end{array}$} \\
\hline & Entre 3 o 4 horas & 77 & 3.77 & 0.960 & \\
\hline & Más de 5 horas & 41 & 4.33 & 0.747 & \\
\hline \multirow{3}{*}{$\begin{array}{c}\text { Redes sociales y } \\
\text { educativas }\end{array}$} & 1 o 2 horas & 70 & 3.78 & 0.763 & \multirow{3}{*}{$\begin{array}{c}\mathrm{F}=8.893 \text { y } \mathrm{p}<.001 \\
\text { favorable al acceso de } \\
\text { más de } 5 \text { horas. }\end{array}$} \\
\hline & Entre 3 o 4 horas & 77 & 4.10 & 0.826 & \\
\hline & Más de 5 horas & 41 & 4.42 & 0.794 & \\
\hline \multirow{3}{*}{ Editores de video } & 1 o 2 horas & 70 & 3.18 & 0.817 & \multirow{3}{*}{$\begin{array}{c}\mathrm{F}=14.963 \text { y } \mathrm{p}<.001 \\
\text { favorable al acceso de } \\
\text { más de } 5 \text { horas y entre } \\
3 \text { o } 4 \text { horas }\end{array}$} \\
\hline & Entre 3 o 4 horas & 77 & 3.62 & 1.019 & \\
\hline & Más de 5 horas & 41 & 4.14 & 0.915 & \\
\hline \multirow{3}{*}{ Editores de imágenes } & 1 o 2 horas & 70 & 3.14 & 0.799 & \multirow{3}{*}{$\begin{array}{l}\mathrm{F}=14.411 \text { y } \mathrm{p}<.001 \\
\text { favorable al acceso de } \\
\text { más de } 5 \text { horas y entre } \\
3 \text { o } 4 \text { horas }\end{array}$} \\
\hline & Entre 3 o 4 horas & 77 & 3.53 & 1.013 & \\
\hline & Más de 5 horas & 41 & 4.07 & 0.884 & \\
\hline \multirow{3}{*}{ Buscadores de imágenes } & 1 o 2 horas & 70 & 3.35 & 0.766 & \multirow{3}{*}{$\begin{array}{c}\mathrm{F}=13.882 \text { y } \mathrm{p}<.001 \\
\text { favorable al acceso de } \\
\text { más de } 5 \text { horas }\end{array}$} \\
\hline & Entre 3 o 4 horas & 77 & 3.68 & 1.017 & \\
\hline & Más de 5 horas & 41 & 4.28 & 0.959 & \\
\hline \multirow{3}{*}{ Buscadores de video } & 1 o 2 horas & 70 & 3.26 & 0.723 & \multirow{3}{*}{$\begin{array}{l}\mathrm{F}=10.226 \text { y } \mathrm{p}<.001 \\
\text { favorable al acceso de } \\
\text { más de } 5 \text { horas y entre } \\
3 \text { o } 4 \text { horas }\end{array}$} \\
\hline & Entre 3 o 4 horas & 77 & 3.70 & 0.965 & \\
\hline & Más de 5 horas & 41 & 4.02 & 1.102 & \\
\hline \multirow{3}{*}{ Buscadores de texto } & 1 o 2 horas & 70 & 3.77 & 0.786 & \multirow{3}{*}{$\begin{array}{c}\mathrm{F}=10.290 \text { y } \mathrm{p}<.001 \\
\text { favorable al acceso de } \\
\text { más de } 5 \text { horas y entre } \\
3 \text { o } 4 \text { horas }\end{array}$} \\
\hline & Entre 3 o 4 horas & 77 & 4.11 & 0.770 & \\
\hline & Más de 5 horas & 41 & 4.42 & 0.698 & \\
\hline \multirow{3}{*}{$\begin{array}{l}\text { Buscadores de } \\
\text { presentaciones }\end{array}$} & 1 o 2 horas & 70 & 3.28 & 0.731 & \multirow{3}{*}{$\begin{array}{c}\mathrm{F}=12.351 \text { y } \mathrm{p}<.001 \\
\text { favorable al acceso de } \\
\text { más de } 5 \text { horas }\end{array}$} \\
\hline & Entre 3 o 4 horas & 77 & 3.57 & 1.043 & \\
\hline & Más de 5 horas & 41 & 4.14 & 0.861 & \\
\hline Wiki & 1 o 2 horas & 70 & 3.53 & 0.744 & $\mathrm{~F}=10.520 \mathrm{y} \mathrm{p}<.001$ \\
\hline
\end{tabular}

Tabla 5. ANOVA del conocimiento de algunas herramientas Web 2.0 en función de las horas al día que se conectan los participantes. 
medio de seis de las once herramientas Web 2.0 de este estudio (existiendo valores significativos en el análisis de Scheffé y Bonferroni entre todas las comparativas), siendo estas:

Los entornos de aprendizaje virtuales [Scheffé: Más de 5 horas (I) - 1 o 2 horas (J) $\mathrm{p}<.001$ y Bonferroni $\mathrm{p}<.001$ y sin significación en la comparativa más de 5 y entre 3 o 4 horas] y los que acceden entre $3 \mathrm{o}$ 4 horas al día [Scheffé: Entre 3 o 4 horas (I) 1 o 2 horas $(J) \mathrm{p}=.008$ y Bonferroni $\mathrm{p}=.006$ ]

Los editores de video [Scheffé: Más de 5 horas (I) - 1 o 2 horas (J) $\mathrm{p}<.001$ y Bonferroni $\mathrm{p}<.001$;Scheffé: Más de 5 horas (I) - Entre 3 o 4 horas $(\mathrm{J}) \mathrm{p}=.013$ y Bonferroni $\mathrm{p}=.009$ ] y entre 3 o 4 horas (Scheffé: Entre 3 o 4 horas $(\mathrm{I})-1 \mathrm{o} 2$ horas $(\mathrm{J}) \mathrm{p}=.013$ y Bonferroni $\mathrm{p}=.010]$.

Los editores de imágenes [Scheffé: Más de 5 horas (I) -1 o 2 horas (J) p<.001 y Bonferroni $p<.001 ;$ Scheffé: Más de 5 horas (I) - Entre 3 o 4 horas (J) $\mathrm{p}=.008$ y Bonferroni $\mathrm{p}=.006]$; mientras que entre 3 o 4 horas al día de conexión [Scheffé: Entre 3 o 4 horas (I) - 1 o 2 horas (J) $\mathrm{p}=.028$ y Bonferroni $\mathrm{p}=.022]$.

Los buscadores de video [Scheffé: Más de 5 horas (I) - 1 o 2 horas $(J) \mathrm{p}<.001$ y Bonferroni $\mathrm{p}<.001$ y sin significación en la comparativa más de 5 horas y entre 3 o 4 horas]; los que acceden entre 3 o 4 horas diarias [Scheffé: Entre 3 o 4 horas (I) - 1 o 2 horas $(\mathrm{J}) \mathrm{p}=.013$ y Bonferroni $\mathrm{p}=.010]$.

La wiki [Scheffé: Más de 5 horas (I) $1 \mathrm{o} 2$ horas $(\mathrm{J}) \mathrm{p}<.001$ y Bonferroni $\mathrm{p}<.001 \mathrm{y}$ sin significación en la comparativa más de 5 horas y entre 3 o 4 horas]; la conexión de entre 3 o 4 horas al día [Scheffé: Entre 3 o 4 horas (I) $-1 \mathrm{o} 2$ horas $(\mathrm{J}) \mathrm{p}=.028$ y Bonferroni $\mathrm{p}=.023]$.

Y, los foros [Scheffé: Más de 5 horas (I) -1 o 2 horas (J) $\mathrm{p}<.001$ y Bonferroni $\mathrm{p}<$
.001; Scheffé: Más de 5 horas (I) - Entre 3 o 4 horas (J) $\mathrm{p}=.023$ y Bonferroni $\mathrm{p}=.018$ ]; $y$ entre 3 o 4 horas al día (Scheffé: Entre 3 o 4 horas (I) -1 o 2 horas $(\mathrm{J}) \mathrm{p}=.043$ y Bonferroni $\mathrm{p}=$ .036].

Además, el alumnado que disfruta de un acceso a Internet de más de 5 horas posee un conocimiento alto de los buscadores de texto en relación a los de 1 o 2 horas al día [Scheffé: Más de 5 horas (I) $-1 \mathrm{o} 2$ horas (J) $\mathrm{p}<.001$ y Bonferroni $\mathrm{p}<.001]$; por el contrario no se aprecian diferencias estadísticas entre las varianzas de más de 5 y entre 3 o 4 horas al día de conectividad. En tanto que, los que gozan de una conexión de entre 3 o 4 horas al día frente a los de 1 o 2 señalan un conocimiento alto al aumentar las horas de acceso [Scheffé: Entre 3 o 4 horas (I) - 1 o 2 horas $(\mathrm{J}) \mathrm{p}=.023$ y Bonferroni $\mathrm{p}=.018$ ] en esta herramienta Web 2.0.

Finalmente, al tratar de establecer diferencias estadísticamente significativas atendiendo al tipo de conexión o red empleada, aplicando para la misma una prueba $\mathrm{t}$ de Student (n.s.= .05), los resultados reflejan datos significativos en todas las herramientas Web 2.0 de este estudio como muestra la Tabla 6.

Los participantes en este estudio que emplean una red pública para conectarse a Internet indican un conocimiento medio - alto de los entornos de aprendizaje virtuales ( $\mathrm{t}=$ 3.278 y $\mathrm{p}=.001,=3.87$ ), de los editores de video $(\mathrm{t}=-3.671$ y $\mathrm{p}<.001,=3.86)$, de los editores de imágenes $(\mathrm{t}=-3.831 \mathrm{y} \mathrm{p}<.001,=$ $3.80)$, de los buscadores de video $(t=-4.579 y$ $\mathrm{p}<.001,=3.94) \mathrm{y}$, de los buscadores de presentaciones $(\mathrm{t}=-4.374 \mathrm{y} \mathrm{p}<.001,=3.92)$ que las personas que utilizan habitualmente una conexión privada.

Asimismo, el cotejo entre medias revela que el conocimiento en $\operatorname{los}$ blogs $(t=-3.478$ y $\mathrm{p}=$ 


\begin{tabular}{|c|c|c|c|c|c|}
\hline $\begin{array}{l}\text { Conocimiento de algunas } \\
\text { herramientas Web } 2.0\end{array}$ & Tipo conexión & $\mathbf{N}$ & Media & $\mathbf{s}$ & $\begin{array}{l}\text { T y p de los grupos } \\
\text { favorables }\end{array}$ \\
\hline \multirow{2}{*}{$\begin{array}{l}\text { Entornos de aprendizaje } \\
\text { virtuales }\end{array}$} & Privada & 109 & 3.43 & 0.919 & \multirow{2}{*}{$\begin{array}{l}\mathrm{T}=-3.278 \text { y } \mathrm{p}=.001 \\
\text { favorable a la pública }\end{array}$} \\
\hline & Pública & 79 & 3.87 & 0.929 & \\
\hline \multirow{2}{*}{ Blogs } & Privada & 109 & 3.58 & 0.908 & \multirow{2}{*}{$\begin{array}{l}\mathrm{T}=-3.478 \text { y } \mathrm{p}=.001 \\
\text { favorable a la pública }\end{array}$} \\
\hline & Pública & 79 & 4.02 & 0.850 & \\
\hline \multirow{2}{*}{ Redes sociales y educativas } & Privada & 109 & 3.90 & 0.848 & \multirow{2}{*}{$\begin{array}{l}\mathrm{T}=-2.968 \text { y } \mathrm{p}=.003 \\
\text { favorable a la pública }\end{array}$} \\
\hline & Pública & 79 & 4.25 & 0.758 & \\
\hline \multirow{2}{*}{ Editores de video } & Privada & 109 & 3.35 & 0.959 & \multirow{2}{*}{$\begin{array}{l}\mathrm{T}=-3.671 \text { y } \mathrm{p}<.001 \\
\text { favorable a la pública }\end{array}$} \\
\hline & Pública & 79 & 3.86 & 0.959 & \\
\hline \multirow{2}{*}{ Editores de imágenes } & Privada & 109 & 3.28 & 0.955 & \multirow{2}{*}{$\begin{array}{l}\mathrm{T}=-3.831 \text { y } \mathrm{p}<.001 \\
\text { favorable a la pública }\end{array}$} \\
\hline & Pública & 79 & 3.80 & 0.915 & \\
\hline \multirow{2}{*}{ Buscadores de imágenes } & Privada & 109 & 3.40 & 0.972 & \multirow{2}{*}{$\begin{array}{l}\mathrm{T}=-5.186 \text { y } \mathrm{p}<.001 \\
\text { favorable a la pública }\end{array}$} \\
\hline & Pública & 79 & 4.08 & 0.839 & \\
\hline \multirow{2}{*}{ Buscadores de video } & Privada & 109 & 3.36 & 0.984 & \multirow{2}{*}{$\begin{array}{l}\mathrm{T}=-4.579 \text { y } \mathrm{p}<.001 \\
\text { favorable a la pública }\end{array}$} \\
\hline & Pública & 79 & 3.94 & 0.812 & \\
\hline \multirow{2}{*}{ Buscadores de texto } & Privada & 109 & 3.93 & 0.792 & \multirow{2}{*}{$\begin{array}{c}\mathrm{T}=-2.517 \text { y } \mathrm{p}=.012 \\
\text { favorable a la pública }\end{array}$} \\
\hline & Pública & 79 & 4.21 & 0.777 & \\
\hline \multirow{2}{*}{ Buscadores de presentaciones } & Privada & 109 & 3.35 & 0.918 & \multirow{2}{*}{$\begin{array}{l}\mathrm{T}=-4.364 \text { y } \mathrm{p}<.001 \\
\text { favorable a la pública }\end{array}$} \\
\hline & Pública & 79 & 3.92 & 0.895 & \\
\hline \multirow{2}{*}{ Wiki } & Privada & 109 & 3.63 & 0.903 & \multirow{2}{*}{$\begin{array}{l}\mathrm{T}=-4.153 \text { y } \mathrm{p}<.001 \\
\text { favorable a la pública }\end{array}$} \\
\hline & Pública & 79 & 4.12 & 0.767 & \\
\hline \multirow{2}{*}{ Foros } & Privada & 109 & 3.55 & 0.871 & \multirow{2}{*}{$\begin{array}{l}\mathrm{T}=-4.651 \text { y } \mathrm{p}<.001 \\
\text { favorable a la pública }\end{array}$} \\
\hline & Pública & 79 & 4.08 & 0.748 & \\
\hline
\end{tabular}

Tabla 6. tStudent del conocimiento de algunas herramientas Web 2.0 en función del tipo de conexión que emplean los participantes.

$.001,=4.02)$, en las redes sociales y educativas $(\mathrm{t}=-2.968$ y $\mathrm{p}=.003,=4.25)$, en los buscadores de imágenes $(\mathrm{t}=-5.186 \mathrm{y} \mathrm{p}<.001,=4.08)$, en los buscadores de texto $(\mathrm{t}=-2.517 \mathrm{y} \mathrm{p}=.012$, $=4.21)$, en la wiki $(\mathrm{t}=-4.153$ y $\mathrm{p}<.001,=4.12)$ $\mathrm{y}$, en los foros $(\mathrm{t}=-4.651 \mathrm{y} \mathrm{p}<.001,=4.08)$, es alto en el alumnado que emplea frecuentemente la red pública frente a los que utilizan la conexión privada.

Píxel-Bit. Revista de Medios y Educación. No 47 Julio 2015. ISSN: 1133-8482. e-ISSN: 2171-7966. doi: http://dx.doi.org/10.12795/pixelbit.2015.i47.03 


\section{Conclusiones y Discusión.}

La discusión del estudio está determinada por las hipótesis planteadas, tanto interrogantes como estadísticas, esta reflexión nos lleva a la cuestión que nos planteábamos (hipótesis de investigación), la cual hacía referencia al grado de conocimiento de un conjunto de 11 herramientas Web 2.0 que se proponían en el estudio, en este sentido, los estudiantes han reflejado un alto conocimiento en las redes sociales y educativas y los procesadores de texto, lo cual constata lo indicado por el Centro Learning \& Performance Technologies (C4LPT) (Hart, 2013), donde se explicita que en el segundo lugar de esta lista se encuentra la herramienta Google Drive/Docs y Twitter, Facebook y Edmodo (primer, noveno y vigesimonoveno lugar, respectivamente) como herramientas de aprendizaje más votadas por los docentes y profesionales del campo educativo; de igual forma, la wiki es señalada en nuestro estudio con un conocimiento bajo y Wikispaces ocupa la posición octogésima en esta lista.

De la batería de once herramientas seis se ven afectadas por la variable curso, en otros términos el alumnado de primero posee un conocimiento más alto de los entornos de aprendizaje virtuales; de los blogs; de las redes sociales y educativas; de los buscadores de video; de los buscadores de texto y de los foros que el resto de estudiantes de los otros cursos del Grado de Educador Social de la Universidad Pablo de Olavide; lo cual no es causa de novedad, dado que como señalan Espuny, González, Lleixà y Gisbert (2011) en el estudio que realizaron el conocimiento en las redes sociales se ve influido por la edad y por lo tanto por los cursos académicos.
La empresa pública comScore dedicada al análisis de medición de la navegación de las personas en el mundo digital, presentó en 2013 un informe sobre el mercado digital español, entre los resultados aportados cabe destacar que en diciembre del 2012 más del $80 \%$ de los teléfonos adquiridos por los españoles entre 15 y 34 años eran smartphones; considerando estos datos, es lógico que en nuestro estudio los resultados obtenidos revelen que el alumnado que emplea el móvil como dispositivo habitual para su conexión a Internet posee un mayor conocimiento del conjunto de las once herramientas Web 2.0 propuestas.

Por último, los estudiantes que emplean una conexión pública y además se conectan con mayor asiduidad (todos los días de la semana y más de cinco horas al día) poseen un mayor conocimiento del conjunto de las once herramientas Web 2.0 propuestas, en especial de las redes sociales y educativas y de los blogs, tal y como señalan Domínguez y Llorente (2009, p.111) «las tecnologías Web 2.0 son recursos muy valiosos en la construcción del conocimiento y en los procesos de aprendizaje social» dado que las mismas desarrollan un aprendizaje más colaborativo, comunicativo y funcional, el cual se ve potenciado por la práctica y experimentalidad frecuente.

López Meneses y Esteban (2008) consideran que: los profesionales de la acción socioeducativa tienen que diseñar y elaborar proyectos de ámbito social que utilicen estas nuevas tecnologías telemáticas para responder de una forma óptima a las demandas y necesidades de la población. También utilizar y reflexionar sobre éstas que, inexorablemente, se perfilan como instrumentos muy interesantes en el ejercicio profesional al ofrecer, entre otras, la 
oportunidad de comunicar, compartir y colaborar entre millones de personas en un mundo interconectado (p. 260).

Al reflexionar sobre esta premisa y considerando los resultados obtenidos en este estudio, los estudiantes del grado de educador social conocen las herramientas Web 2.0 lo cual facilitará su aplicación en la intervención socioeducativa formal a través de la elaboración y diseño de proyectos, programas y planes que favorezcan las relaciones en los centros educativos con estas herramientas.

En este sentido, esta investigación complementa a la experiencia didáctica realizada por López Meneses, Llorent y Fernández Márquez (2013) y a la investigación de corte cualitativo basada en un proyecto de innovación de López Meneses, Fernández Sánchez, Cobos y Pedrero (2012) en las cuales, entre otros aspectos, se considera el conocimiento de las herramientas web 2.0 en los estudiantes del Grado de Educador Social como los blogs o las wikis.

En conclusión, partiendo de que existen pocos referentes de investigación sobre el tema tratado en este artículo, el estudio presentado sobre el grado de conocimiento que poseen los estudiantes del Grado de Educador Social sobre determinadas herramientas Web 2.0 proporciona datos para optimar y perfeccionar el proceso de enseñanza - aprendizaje y futuras innovaciones educativas.

\section{Referencias bibliográficas.}

ANECA. (2005). Libro Blanco de Grado en Pedagogía y Educación Social (vol. 1). Madrid: Agencia Nacional de Evaluación de la Calidad y Acreditación.
Cabero, J. (2009). Educación 2.0. ¿Marca, moda o nueva visión de la educación?. En C. Castaño (coord). Web 2.0. El uso de la Web en la sociedad del conocimiento. Investigación e implicaciones educativas (pp. 13-34). Venezuela: Universidad Metropolitana. Recuperado de: http:// tecnologiaedu.us.es/tecnoedu/images/ stories/castanio20.pdf

Comscore (2013). Spain Digital Future in Focus 2013. Recuperado de: http:// www.comscore.com/esl/Insights/ Presentations-and-Whitepapers/2013/2013Spain-Digital-Future-in-Focus

Domínguez, G. \& Llorente, M.C. (2009). La educación social y la web 2.0: Nuevos espacios de innovación e interacción social en el espacio europeo de educación superior. Pixel Bit. Revista de Medios y Educación, 35, 105-114. Recuperado de: http:// www.sav.us.es/pixelbit/pixelbit/articulos/n35/ 9.html

Espuny, C., González, J., Lleixà, M. \& Gisbert, M. (2011). Actitudes y expectativas del uso educativo de las redes sociales en los alumnos universitarios. RUSC. Revista de Universidad y Sociedad del Conocimiento, 8 (1). 171-185. Recuperado de:http:// rusc.uoc.edu/index.php/rusc/article/view/ v8n1-espuny-gonzalez-lleixa-gisbert/v8n1espuny-gonzalez-lleixa-gisbert

Esteve, F. (2009). Bolonia y las TIC: de la docencia 1.0 al aprendizaje 2.0. La cuestión universitaria, 5, 59- 68. Recuperado de: http:/ /dialnet.unirioja.es/servlet/ articulo? codigo $=2950729$

Freire, J. (2007). Los retos y oportunidades de la web 2.0 para las universidades. En Polo, F. \& Jiménez, R. La gran guía de los blogs 2008. Colección Planta 29 (pp. 82-90). Barcelona: Cobre Ediciones. 
Gómez Gutiérrez, J.L. (2000). Intervención socioeducativa en el medio escolar: papel del educador social escolar. Indivisa. Boletín de Estudios e Investigación, 1, 59-76. Recuperado de: http://www.redalyc.org/ articulo.oa?id=77100103

Grodecka, K., Pata, K. \& Väljataga, T. (2008). Web 2.0 and education. En Grodecka, K., Wild,F. \& Kieslinger,B. (Eds.). How to use social software in Higher Education (pp. 10-12). Polonia, Cracovia: Wydawnictwo Naukowe Akapit.

Hart, J. (2013). The Top 100 Tools for Learning List. Centre for Learning \& Performance Technologies.(C4PLT). Recuperado de: http://c4lpt.co.uk/ top100tools/

Herrera, D. (2010). Un acercamiento a la situación formativa de los educadores y educadoras sociales. Revista de Educación, 353, 641-666. Recuperado de: http:// www.mecd.gob.es/dctm/revista-dee d u c a c i on/articulos re $353 /$ re35324.pdf?documentId=0901e72b812048c5

López Meneses, E. \& Esteban, M. (2008). La educación social y las nuevas tecnologías de la información y la comunicación: nuevos espacios en la construcción e intervención socioeducativa. Revista Latinoamericana de Estudios Educativos, XXXVIII (1-2), 255-287. Recuperado de: http://www.redalyc.org/pdf/ 270/27012437010.pdf

López Meneses, E., Fernández Sánchez, M. R., Cobos, D. \& Pedrero, E. (2012). Implicaciones de las Tics en el ámbito socio - educativo y de servicios sociales: una experiencia universitaria de innovación y desarrollo docente con tecnologías 2.0. Campo Abierto,31 (2), 11-35. Recuperado de: http://redined.mecd.gob.es/xmlui/handle/ 11162/96584?locale-attribute=en
López Meneses, E., Llorent, V.J. \& Fernández Márquez, E. (2013). Experiencia universitaria sobre las funciones del educador/a social con tecnologías 2.0. Edutec. Revista Electrónica de Tecnología Educativa, 43, 1-17. Recuperado de: http:// edutec.rediris.es/Revelec2/Revelec43/pdf/ Edutec-e_n43-Lopez_Llorent_Fernandez.pdf Martínez, A. (2004). La enseñanza y el aprendizaje de las tecnologías de la información y la comunicación en la intervención socioeducativa. Cuadernos de trabajo social, 17, 237-253. Recuperado de: http://revistas.ucm.es/index.php/CUTS/ article/view/CUTS0404110237A/7615

Mateo, J. (2012). La investigación ex postfacto. En R. Bisquerra (coord.). Metodología de la investigación educativa (pp. 195-229) ( $3^{\mathrm{a}}$. ed.). Madrid: La Muralla.

Morales, P. (2012). Estadística aplicada a las Ciencias Sociales. Tamaño necesario de la muestra: ¿Cuántos sujetos necesitamos? Madrid: Universidad Pontificia Comillas. Recuperado de: http://web.upcomillas.es/ personal/peter/investigacion/ Tama\%F1omuestra.pdf

Morales, P. (2011). Análisis de varianza para varias muestras independientes. Madrid: Universidad Pontificia Comillas. Recuperado de: http://web.upcomillas.es/ personal/peter/analis isdevarianza/ ANOVAIndependientes.pdf

Sevillano, M.L. (2010). Sociedad de la información-sociedad del conocimiento: relaciones y convergencia. En M.L. Sevillano (coord.). Nuevas tecnologías en educación social (pp. 1-28). Madrid: Mc Graw Hill.

Fecha de recepción: 14-08-2014 Fecha de evaluación: 17-09-2014

Fecha de aceptación: 20-10-2014 